\title{
Acné en el recién nacido
}

\author{
Juan A. Godínez-Chaparro ${ }^{1 *}$ y Helena Vidaurri-De la Cruz ${ }^{2}$
}

${ }^{1}$ Servicio de Dermatología Pediátrica, Unidad Médica de Alta Especialidad Hospital General Dr. Gaudencio González Garza, Centro Médico Nacional la Raza, Instituto Mexicano del Seguro Social; ${ }^{2}$ Servicio de Dermatología Pediátrica, Hospital General de México Dr. Eduardo Liceaga, Secretaría de Salud. Ciudad de México, México

\section{Resumen}

El acné neonatal es una dermatosis transitoria que ocurre entre la segunda y la cuarta semanas de vida en uno de cada cinco niños. Es más frecuente en los varones, con una relación de sexo masculino-femenino de 4.5:1. Las manifestaciones clínicas incluyen comedones abiertos y cerrados que pueden progresar a lesiones inflamatorias como pápulas, pústulas eritematosas y, en casos raros, nódulos y quistes. Las zonas afectadas incluyen la frente, las mejillas, el mentón y los párpados, y en algunas ocasiones puede extenderse a la piel cabelluda, el cuello y el tronco. Ocurre por la mayor producción de andrógenos placentarios y neonatales (de origen suprarrenal en ambos sexos y de origen testicular en los varones), que provoca hipertrofia de las glándulas sebáceas y mayor producción de sebo. La mayoría de los casos son leves y autolimitados. Cuando el acné neonatal es grave y prolongado, se debe considerar la posibilidad de hiperandrogenismo, cuyas causas más frecuentes en esta edad son la hiperplasia suprarrenal congénita y los tumores, adrenales o gonadales, productores de andrógenos. El diagnóstico del acné neonatal es clínico: se debe distinguir de la pustulosis cefálica neonatal, otras dermatosis neonatales vesiculopustulares, enfermedades infecciosas y reacciones acneiformes. Habitualmente, un dermolimpiador suave y agua resultan suficientes para su manejo. Las lesiones obstructivas (comedones abiertos y cerrados) pueden requerir retinoides tópicos o ácido azelaico al 20\%, y las lesiones inflamatorias, algunos antibióticos tópicos.

Palabras clave: Acné neonatal. Acné neonatorum. Acné del recién nacido. Acné.

\section{Acne in the newborn}

\section{Abstract}

Neonatal acne (NA) is a transitory dermatosis that occurs between the second and fourth weeks of life in $20 \%$ of children. This condition is more frequent in males, with a male-female ratio of 4.5:1. Present primary skin lesions are open and closed comedones which can evolve into papules, erythematous pustules and, in rare cases, nodules and cysts. NA topography includes the forehead, cheeks, chin, and eyelids, but occasionally it spreads to the scalp, neck, and trunk. NA occurs due to an elevated production of placental and neonatal androgens (of adrenal origin in both sexes and of testicular origin in males) which cause enlargement of the sebaceous glands and increases the production of sebum. Most cases are mild and transient, but if NA is severe and long-lasting, clinical and paraclinical examination will be necessary to find congenital adrenal hyperplasia or a virilizing tumor of adrenal or gonadal origin. The diagnosis of NA is clinical; its main differential diagnoses

\section{Correspondencia:}

*Juan A. Godínez-Chaparro

E-mail: alberto.godinezch@gmail.com (http://creativecommons.org/licenses/by-nc-nd/4.0/).
Fecha de recepción: 27-10-2020

Fecha de aceptación: 29-12-2020

DOI: 10.24875/BMHIM.20000327
Disponible en internet: 17-09-2021 Bol Med Hosp Infant Mex. 2021;78(5):443-449

www.bmhim.com 
are neonatal cephalic pustulosis, other neonatal vesiculopustular dermatoses, infectious diseases, and acneiform reactions. The resolution of NA is spontaneous. In most cases, the use of a mild dermal cleanser and water will be sufficient. For comedogenic lesions (open and closed comedones), topical retinoids or $20 \%$ azelaic acid may be used, as well as some topical antibiotics for inflammatory lesions.

Keywords: Neonatal acne. Neonatorum acne. Newborn acne. Acne.

\section{Introducción}

El recién nacido (RN) atraviesa por un periodo de adaptación cuando deja el medio amniótico y pasa al medio aéreo. Durante esta etapa, aparecen algunas dermatosis benignas y transitorias que se clasifican de acuerdo con su morfología. Dentro de las pustulosas se encuentran el eritema tóxico, la melanosis pustulosa transitoria del RN, la miliaria y el acné neonatal' ${ }^{1}$.

En 1913, Kraus describió esta afección, que puede aparecer desde el nacimiento, aunque suele iniciar entre la segunda y la cuarta semanas de vida en uno de cada cinco recién nacidos ${ }^{2}$. Es 4.5 veces más frecuente en el sexo masculino $0^{3-5}$. Se caracteriza por lesiones inflamatorias y obstructivas en la zona facial. Lo habitual es que sea leve. Sin embargo, algunos casos presentan lesiones inflamatorias más persistentes ${ }^{6,7}$.

De acuerdo con el Consenso Ibero-Latinoamericano del año 2014 que realizaron el Grupo Ibero-Latinoamericano de Estudio del Acné (GILEA) y el Colegio Ibero-LatinoAmericano de Dermatología (CILAD), el acné se puede clasificar por grupos de edad en acné neonatal, del lactante, infantil, preadolescente, adolescente y del adulto ${ }^{8}$. Existe cierto grado de controversia con respecto a la nomenclatura del acné neonatal y de lo que se ha denominado pustulosis cefálica neonatal. El acné neonatal ocurre por la mayor producción de andrógenos placentarios y neonatales que provoca hipertrofia de las glándulas sebáceas y mayor producción de sebo. Estos cambios favorecen la aparición de comedones abiertos y cerrados que pueden progresar a lesiones inflamatorias, como pápulas, pústulas eritematosas y, en casos raros, nódulos y quistes. La pustulosis cefálica neonatal parece ocurrir en respuesta a la colonización por Malassezia; en este padecimiento no aparecen comedones abiertos y cerrados como en el acné neonatal ${ }^{9}$.

\section{Patogenia}

Existen diversos factores relacionados con la formación del acné neonatal. Las glándulas sebáceas fetales son muy similares a las del adulto, pues son sensibles al efecto de los andrógenos, sean de origen placentario, adrenal o gonadal ${ }^{10-14}$. Durante la infancia, antes de la adrenarca, las glándulas son pequeñas y producen poco sebo. Sin embargo, con los cambios hormonales de la pubertad, estas glándulas se agrandan de nuevo y se incrementa la producción de sebo (Figura 1) 15 .

Los RN presentan valores elevados de hormona luteinizante $y$, posteriormente, también valores altos de testosterona debido a la inmadurez del mecanismo de retroalimentación gonadal ${ }^{16}$. En los varones de 1 mes de edad, la testosterona alcanza valores semejantes a los puberales y disminuye a cifras prepuberales a los 6 meses de edad ${ }^{14,17}$. Tanto en el sexo masculino como en el femenino se producen andrógenos débiles en la corteza suprarrenal: en la zona reticular se sintetizan dehidroepiandrosterona, su forma sulfatada y androstenediona ${ }^{14,18}$. Hacia el año de edad, la glándula suprarrenal paulatinamente adquiere las proporciones de la

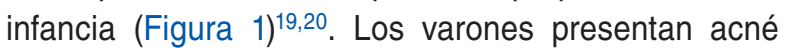
neonatal con más frecuencia porque sus glándulas sebáceas reciben estimulación androgénica desde la suprarrenal y el testículo ${ }^{20}$.

En la fisiología de los andrógenos, la dehidroepiandrosterona se metaboliza a $\Delta 4$-androstenediona por medio de la $3 \beta$-hidroxiesteroide deshidrogenasa tipo 2 . Posteriormente, con la ayuda la $17 \beta$-hidroxiesteroide deshidrogenasa tipo 3 se sintetiza testosterona. Estas enzimas esteroidogénicas se han localizado en las glándulas sebáceas. Para terminar, la conversión intracelular de testosterona a $5 \alpha$-dihidrotestosterona se produce por las isoenzimas 1,2 y 3 de la $5 \alpha$-reductasa ${ }^{13,21}$. La $5 \alpha$-dihidrotestosterona es mucho más potente que la testosterona y los andrógenos suprarrenales ${ }^{13}$. Las isoenzimas 1 y 2 de la $5 \alpha$-reductasa se expresan en la piel y el folículo pilosebáceo de manera transitoria en el RN de término y de forma permanente en el adolescente. Esta expresión regulada podría explicar por qué el acné neonatal solo ocurre en $\mathrm{RN}$ de término mayores de 37 semanas de gestación, y no en prematuros, a pesar de haber una mayor secreción andrógena en ellos ${ }^{14,22}$.

La traducción clínica del estímulo androgénico es una marcada secreción de sebo, equiparable a la de un adulto joven, que se mantiene constante durante todo 


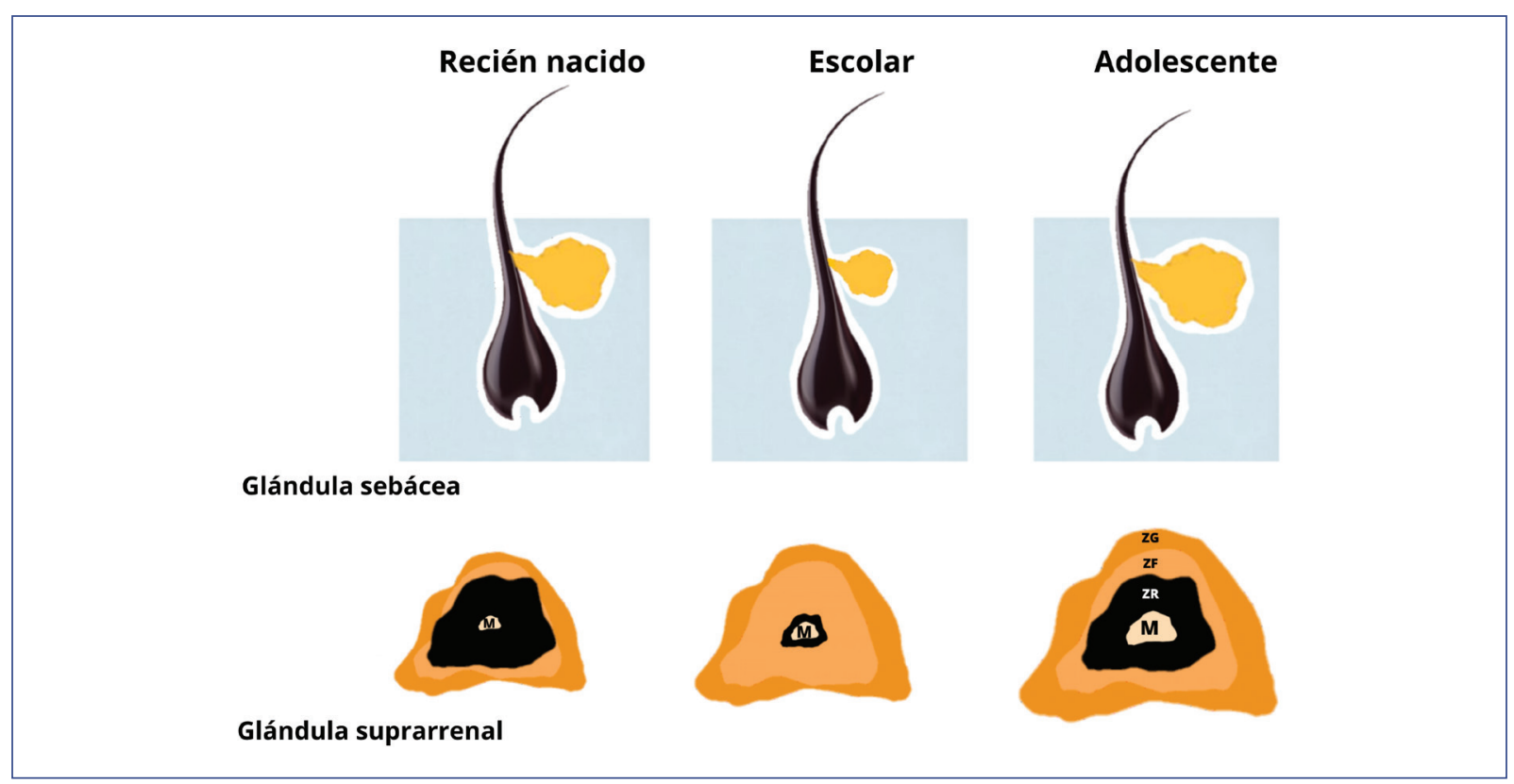

Figura 1. La zona reticular de la glándula suprarrenal del feto y del recién nacido produce altas cantidades de andrógenos. Durante la infancia cesa dicha producción y de nuevo aumenta en la pubertad. En virtud de que las glándulas sebáceas son sensibles a los andrógenos séricos, su tamaño y función se relacionan con los valores de estas hormonas. M: médula; ZR: zona reticular; ZF: zona fascicular; ZG: zona glomerular.

el primer mes. Esta secreción disminuye de manera paulatina a partir del segundo mes y hasta el final del primer año ${ }^{23}$.

Para el funcionamiento adecuado de la barrera cutánea se requiere una proporción adecuada de ácidos grasos, ceramidas y colesterol. Los lípidos de la epidermis neonatal son muy similares a los maternos ${ }^{13,24}$. En la epidermis de las madres de pacientes con acné neonatal se ha encontrado que la cantidad de ácidos grasos de cadena larga, ceramidas, triglicéridos y diglicéridos es menor que en la epidermis de poblaciones sin acné neonatal. Estas deficiencias disminuyen la función de la barrera cutánea, lo que puede favorecer la aparición del acné24. Los altos valores de linoleato en el sebo neonatal podrían ser un factor protector ${ }^{13}$.

Algunos autores no están convencidos de la existencia del acné neonatal, ya que es posible que muchos de estos casos sean en realidad una pustulosis cefálica neonatal que aparece en respuesta a la colonización por Malassezia ${ }^{25}$ en el 30-52\% de los RN entre la segunda y la cuarta semanas de vida ${ }^{26,27}$. En esta dermatosis los pacientes presentan pápulas y pústulas monomorfas eritematosas, pero no cursan con comedones abiertos ni cerrados (característicos del acné neonatal). Por otro lado, algunos autores sugieren que pueden coexistir ambos fenotipos ${ }^{5}$.

\section{Cuadro clínico}

Las lesiones pueden estar presentes desde el nacimiento, aunque lo más común es que aparezcan entre la segunda y la sexta semanas de vida. Estas lesiones se curan de manera espontánea sin dejar cicatrices a partir de la cuarta semana de vida, aunque en algunos casos pueden persistir durante 3-6 meses $^{28}$.

Las zonas afectadas por el acné neonatal incluyen la frente, las mejillas, el mentón y los párpados; en ocasiones, se extiende a la piel cabelluda, el cuello y el tronco a nivel del pecho y la espalda ${ }^{5,29}$. Las lesiones elementales son comedones abiertos y cerrados (lesiones obstructivas), que pueden progresar a pápulas y pústulas eritematosas (Figura 2) 4,30. La mayoría de los casos son leves y transitorios. En algunos pacientes pueden aparecer nódulos y quistes. En estos casos, se denomina acné infantil al que persiste más allá del periodo neonatal ${ }^{5}$.

En un grupo de 22 RN con acné neonatal, en Grecia, se encontró que el $81.8 \%$ eran de sexo masculino. La dermatosis inició a las 3 semanas y duró 4 meses. El $72.7 \%$ presentaron pápulas y pústulas, el $22.7 \%$ comedones y el $4.5 \%$ lesiones inflamatorias y quistes. La zona más afectada fue la mejilla (81.8\%). Solo en el $13.6 \%$ de los pacientes se reportó historia familiar de acné $^{3}$. 


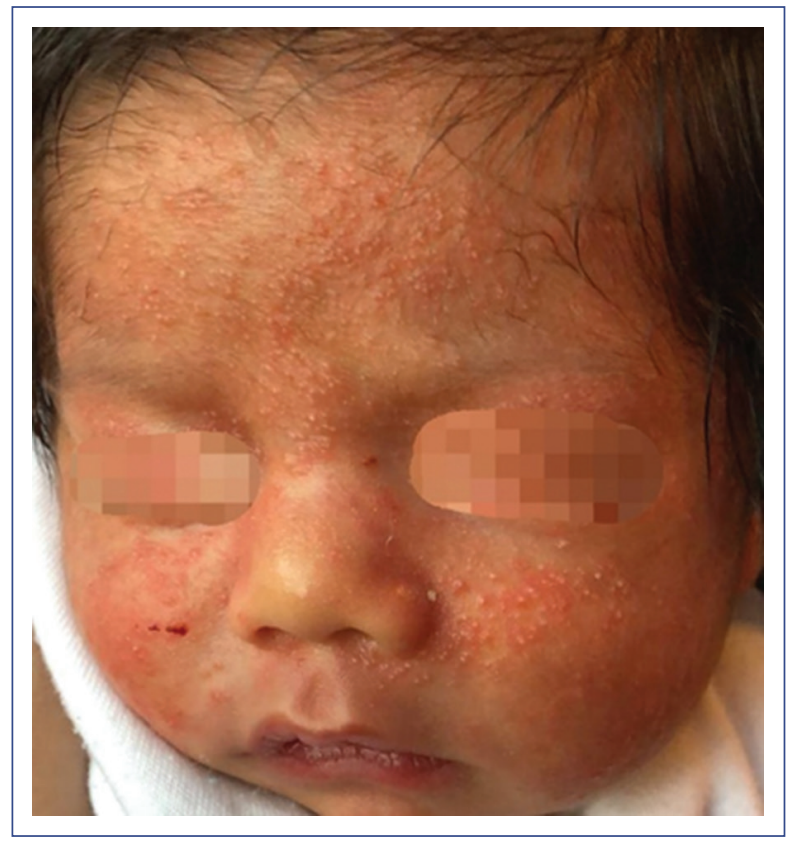

Figura 2. Varón de 18 días de vida. Se observan pápulas y pústulas en las mejillas, la frente y la nariz. (Archivo clínico de la Dra. Helena Vidaurri.)

En una serie de $26 \mathrm{RN}$, en Arabia Saudita, se encontró que el $30.8 \%$ presentaron comedones, el $15.3 \%$ pápulas y pústulas, y el $53.4 \%$ una combinación de pápulas, pústulas y quistes. La media de edad de inicio fue a las 1.19 semanas y la duración fue de 1 mes. Se reportaron antecedentes familiares positivos para acné en la mitad de los pacientes ${ }^{31}$.

Algunos factores genéticos y ambientales, así como las variaciones culturales para el cuidado de la piel, pueden influir en la presentación clínica del acné neonatal.

\section{Diagnóstico}

El diagnóstico es básicamente clínico. En muy pocos casos se requieren exámenes paraclínicos. En las ocasiones en que se ha realizado un análisis histológico, ha sido únicamente con fines de investigación y se han encontrado glándulas sebáceas hiperplásicas con tapones de queratina. De forma ocasional, las unidades pilosebáceas pueden romperse y generar inflamación ${ }^{3}$.

El diagnóstico diferencial debe realizarse con dermatosis vesiculopustulares infecciosas, no infecciosas y acneiformes (Tabla 1) 4,27,32-39. Las dermatosis pustulosas de origen infeccioso incluyen la foliculitis bacteriana, la sífilis secundaria, el herpes simple, la varicela y la colonización por especies de Malassezia ${ }^{40}$. Entre las no infecciosas se encuentran la pustulosis cefálica neonatal, el eritema tóxico, la melanosis pustulosa transitoria del RN, la hiperplasia sebácea y la miliaria rubra. El principal diagnóstico diferencial es con la pustulosis cefálica neonatal. Sin embargo, en esta dermatosis transitoria, la etiología se ha asociado al crecimiento excesivo de levaduras lipófilas de Malassezia spp. (principalmente M. sympodialis, M. furfur y M. globosa). Las lesiones usualmente son pápulas y pústulas monomorfas y eritematosas, sin presencia de comedones y con buena respuesta a los azoles tópicos como el ketoconazol ${ }^{4,27,32}$.

Las dermatosis acneiformes, en las que la lesión elemental más común es el comedón, incluyen las siguientes variantes:

- El acné pomada, debido a la aplicación de aceites, cremas, pomadas 0 ungüentos ${ }^{41}$.

- Las dermatosis acneiformes secundarias al uso de medicamentos durante el embarazo, como litio, halógenos sistémicos, hidantoína y esteroides ${ }^{7,28,42}$.

- El cloracné, que se presenta después de la exposición a hidrocarburos clorados con una afectación preferente del área temporal ${ }^{43,44}$.

- La coexistencia de luteoma virilizante y embarazo ${ }^{7,28}$. Cuando el acné neonatal persiste, es decir, si su duración es mayor de 4 meses $^{3}$, y cuando es grave (presenta nódulos y quistes), se debe buscar una causa endocrina de virilización: hiperplasia suprarrenal congénita o tumores (adrenales o gonadales) productores de andrógenos. Algunos signos clínicos de estos trastornos son la virilización de los genitales y cambios en el crecimiento y el desarrollo (Figura 3). Además, algunos pacientes con acné neonatal podrían presentar acné grave en la adolescencia ${ }^{4,5,30}$.

\section{Tratamiento}

El acné neonatal es una dermatosis leve y autolimitada que dura de 4 semanas a 4 meses, y no deja cicatrices. Aunque es raro, si persiste hasta los 12 meses, se le denomina acné infantil ${ }^{45}$. En la mayor parte de los casos, las medidas habituales de higiene de la piel son suficientes para la resolución gradual de la dermatosis ${ }^{46}$. Por ejemplo, en la serie de Katsambas, et al. ${ }^{3}$, el $81.8 \%$ de los pacientes solo necesitaron limpieza diaria con agua y jabón. En el estudio de Alakloby et al. ${ }^{31}$, todos los pacientes se manejaron con aseo y limpieza diaria. Algunos pacientes con lesiones obstructivas pueden requerir retinoides tópicos, como tretinoína (al 0.025-0.05\%) o crema con ácido azelaico (al 
Tabla 1. Diagnósticos diferenciales del acné neonatal frente a otras dermatosis transitorias del recién nacido

\begin{tabular}{|c|c|c|c|c|c|c|}
\hline Fenotipo & Acné neonatal & $\begin{array}{l}\text { Pustulosis } \\
\text { cefálica } \\
\text { neonatal }\end{array}$ & Eritema tóxico & $\begin{array}{l}\text { Melanosis } \\
\text { pustulosa } \\
\text { transitoria del RN }\end{array}$ & $\begin{array}{l}\text { Miliaria rubra/ } \\
\text { pustulosa }\end{array}$ & $\begin{array}{l}\text { Hiperplasia } \\
\text { sebácea }\end{array}$ \\
\hline Referencias & & $\begin{array}{l}\text { Antoniou, } \\
\text { et al. }{ }^{4} \text {, } \\
\text { Bernier, } \\
\text { et al.. } \\
\text { Rapelanoro, } \\
\text { et al. }{ }^{32} \text {, } \\
\text { Yañes-Díaz, } \\
\text { et al. }{ }^{33}\end{array}$ & $\begin{array}{l}\text { Antoniou, et al. }{ }^{4}, \\
\text { Reginatto, et al. }{ }^{34,} \\
\text { Hulsmann y } \\
\text { Oranje } \\
\text { Hernández }^{35}\end{array}$ & $\begin{array}{l}\text { Reginatto, et al. }{ }^{34} \text {, } \\
\text { Hulsmannn y } \\
\text { Oranje }{ }^{35} \text {, } \\
\text { Hernández }^{36}\end{array}$ & $\begin{array}{l}\text { Antoniou, et al. }{ }^{4} \text {, } \\
\text { Reginatto, } \\
\text { et al.. } \\
\text { Hernández }{ }^{36} \text {, } \\
\text { Guerra, et al. }{ }^{37}\end{array}$ & $\begin{array}{l}\text { Krüger, } \\
\text { et al. }{ }^{38,} \\
\text { Eichenfield, } \\
\text { et al. }{ }^{39}\end{array}$ \\
\hline Etiología & $\begin{array}{l}\text { Andrógenos } \\
\text { maternos y } \\
\text { neonatales. } \\
\text { Incremento en } \\
\text { la producción } \\
\text { sebácea }\end{array}$ & $\begin{array}{l}\text { Crecimiento } \\
\text { excesivo de } \\
\text { levaduras } \\
\text { lipófilas de } \\
\text { Malassezia spp. } \\
\text { (M. sympodialis, } \\
\text { M. furfur y } \\
\text { M. globosa) }\end{array}$ & $\begin{array}{l}\text { Probablemente } \\
\text { reacción } \\
\text { inflamatoria a la } \\
\text { colonización } \\
\text { microbiana, } \\
\text { absorción de } \\
\text { enterotoxinas o } \\
\text { reacción alérgica a } \\
\text { agentes ambientales } \\
\text { o placentarios }\end{array}$ & $\begin{array}{l}\text { Desconocido. } \\
\text { Se ha propuesto } \\
\text { como } \\
\text { manifestación } \\
\text { precoz de eritema } \\
\text { tóxico neonatal }\end{array}$ & $\begin{array}{l}\text { Obstrucción del } \\
\text { conducto de la } \\
\text { glándula ecrina. } \\
\text { Predomina en } \\
\text { verano, } \\
\text { periodos } \\
\text { febriles o } \\
\text { exceso de ropa }\end{array}$ & $\begin{array}{l}\text { Estimulación } \\
\text { androgénica } \\
\text { de las } \\
\text { glándulas } \\
\text { sebáceas }\end{array}$ \\
\hline Epidemiología & $\begin{array}{l}\text { Relación M/F } \\
\text { 4.5:1 }\end{array}$ & $\begin{array}{l}\text { RN de peso } \\
\text { bajo, } \\
\text { hospitalización } \\
\text { prolongada en } \\
\text { UCI }\end{array}$ & $\begin{array}{l}\text { Masculino } \\
\text { RN de término } \\
\text { Peso }>2500 \mathrm{~g} \\
\text { Apgar }>8\end{array}$ & $\begin{array}{l}\text { Masculino y } \\
\text { femenino. } \\
\text { Ascendencia } \\
\text { africana }\end{array}$ & $\begin{array}{l}\text { Masculino y } \\
\text { femenino }\end{array}$ & $\begin{array}{l}\text { Relación M/F } \\
1: 1 \\
\text { RN de } \\
\text { término }\end{array}$ \\
\hline Prevalencia & $20 \%$ & $10-66 \%$ & $\begin{array}{l}16-40 \% \\
30-70 \%\end{array}$ & $\begin{array}{l}3.4 \% \\
1-4 \%\end{array}$ & $30 \%$ & $50-66.6 \%$ \\
\hline Edad & $2-4$ semanas & $\begin{array}{l}5 \text { días a } 3 \\
\text { semanas }\end{array}$ & $24-72$ horas de vida & $\begin{array}{l}\text { Nacimiento a } 48 \\
\text { horas }\end{array}$ & 1 a 3 semanas & Nacimiento \\
\hline Topografía & $\begin{array}{l}\text { Cabeza: frente y } \\
\text { mejillas, } \\
\text { mentón, } \\
\text { párpados. } \\
\text { Ocasionalmente } \\
\text { piel cabelluda, } \\
\text { cuello, pecho y } \\
\text { espalda }\end{array}$ & $\begin{array}{l}\text { Cara: frente, } \\
\text { párpados, } \\
\text { mejillas y } \\
\text { mentón. } \\
\text { Piel cabelluda, } \\
\text { cuello, tronco } \\
\text { anterior y } \\
\text { posterior }\end{array}$ & $\begin{array}{l}\text { Cara, pecho, tronco } \\
\text { y extremidades. } \\
\text { Respeta palmas y } \\
\text { plantas }\end{array}$ & $\begin{array}{l}\text { Frente, barbilla, } \\
\text { cuello, parte } \\
\text { superior de pecho, } \\
\text { región lumbar } \\
\text { baja, muslos, } \\
\text { glúteos, abdomen, } \\
\text { palmas y plantas }\end{array}$ & $\begin{array}{l}\text { Zonas de flexión } \\
\text { y regiones } \\
\text { cubiertas del } \\
\text { cuerpo, como el } \\
\text { tronco }\end{array}$ & $\begin{array}{l}\text { Frente, nariz, } \\
\text { mejillas y } \\
\text { labio superior }\end{array}$ \\
\hline Morfología & $\begin{array}{l}\text { Comedones } \\
\text { abiertos y } \\
\text { cerrados, } \\
\text { pápulas y } \\
\text { pústulas } \\
\text { eritematosas }\end{array}$ & $\begin{array}{l}\text { Pápulas y } \\
\text { pústulas } \\
\text { monomorfas } \\
\text { eritematosas }\end{array}$ & $\begin{array}{l}\text { Máculas, pápulas, } \\
\text { vesículas y pústulas } \\
\text { con una base } \\
\text { eritematoedematosa }\end{array}$ & $\begin{array}{l}\text { Vesiculopústulas } \\
\text { superficiales y } \\
\text { flácidas que dejan } \\
\text { un collar de } \\
\text { escamas y máculas } \\
\text { hiperpigmentadas }\end{array}$ & $\begin{array}{l}\text { Pápulas, } \\
\text { vesículas y } \\
\text { pústulas } \\
\text { eritematosas, } \\
\text { pruriginosas }\end{array}$ & $\begin{array}{l}\text { Pápulas de } \\
\text { color blanco } \\
\text { amarillento } \\
\text { puntiformes } \\
\text { sin eritema ni } \\
\text { reacción } \\
\text { inflamatoria }\end{array}$ \\
\hline Diagnóstico & $\begin{array}{l}\text { Clínico. } \\
\text { Si es grave o } \\
\text { prolongado, } \\
\text { buscar fuente } \\
\text { de andrógenos } \\
\text { (adrenal o } \\
\text { gonadal) }\end{array}$ & $\begin{array}{l}\mathrm{KOH} \\
\text { aislamiento de } \\
\text { esporas y } \\
\text { levaduras de } \\
\text { Malassezia } \\
\text { spp. }\end{array}$ & $\begin{array}{l}\text { Eosinofilia (15\%) } \\
\text { Tinción de Tzanck o } \\
\text { Gram con } \\
\text { abundantes } \\
\text { eosinófilos y } \\
\text { escasos neutrófilos }\end{array}$ & $\begin{array}{l}\text { Contenido de } \\
\text { pústulas: } \\
\text { polimorfonucleares } \\
\text { neutrófilos, } \\
\text { eosinófilos } \\
\text { aislados }\end{array}$ & $\begin{array}{l}\text { En la citología } \\
\text { se observan } \\
\text { linfocitos } \\
\text { Aislamiento de } \\
\text { Staphylococcus } \\
\text { aureus en } 29.4 \%\end{array}$ & Clínico \\
\hline Tratamiento & $\begin{array}{l}\text { Autolimitado. } \\
\text { Lesiones } \\
\text { crónicas e } \\
\text { inflamatorias: } \\
\text { tretinoína } \\
0.025-0.05 \% \text {, } \\
\text { peróxido de } \\
\text { benzoilo, } \\
\text { clindamicina } \\
\text { tópicos }\end{array}$ & $\begin{array}{l}\text { Antifúngicos } \\
\text { azólicos como } \\
\text { ketoconazol } \\
\text { tópico } \\
2 \text { veces/día } \\
\text { por } 1 \text { mes }\end{array}$ & $\begin{array}{l}\text { Autolimitado en } 24 \\
\text { a } 72 \text { horas }\end{array}$ & $\begin{array}{l}\text { Autolimitado en } \\
\text { 3-4 semanas }\end{array}$ & $\begin{array}{l}\text { Uso de ropa } \\
\text { ligera y } \\
\text { ambientes } \\
\text { frescos } \\
\text { Secantes }\end{array}$ & $\begin{array}{l}\text { Autolimitado. } \\
\text { Rápida } \\
\text { remisión }\end{array}$ \\
\hline
\end{tabular}

\footnotetext{
F: femenino; KOH: hidróxido de potasio; M: masculino; RN: recién nacido; UCl: unidad de cuidados intensivos.
} 


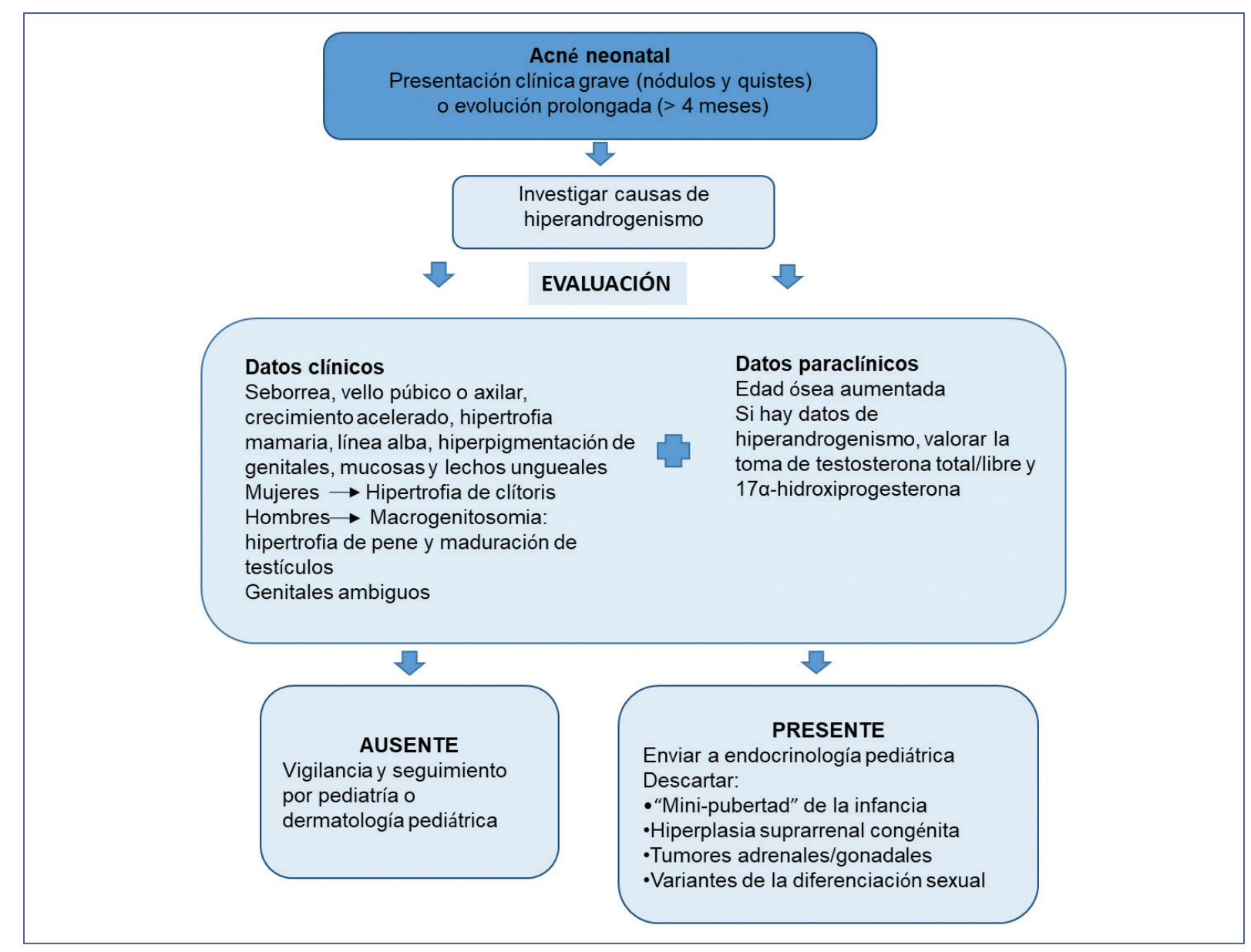

Figura 3. Abordaje del acné neonatal grave o prolongado.

20\%). Para las lesiones inflamatorias se pueden utilizar antibióticos tópicos, como eritromicina (al 2\%) y peróxido de benzoilo (al 2\%) $)^{3,40,47}$. En el estudio de Katsambas, et al. ${ }^{3}$ solo el $18.1 \%$ de los pacientes requirieron el uso de algún antibiótico tópico.

Si el acné empeora o es recalcitrante al tratamiento, se debe referir al dermatólogo pediatra y al endocrinólogo pediatra para buscar causas endocrinas de virilización y disminuir el riesgo de cicatrices ${ }^{40,47}$.

Hasta ahora no se ha utilizado isotretinoína sistémica para el tratamiento del acné neonatal, pero sí se ha empleado en niños mayores con acné infantil que presentaron lesiones inflamatorias recalcitrantes al tratamiento y formación de cicatrices. Dichas secuelas se han reportado en el $17 \%$ de los pacientes. La información sobre el tratamiento sistémico es limitada y solo existen pequeñas series de casos que describen su eficacia. Las dosis de isotretinoína empleadas en la etapa infantil son bajas $(0.2-0.5 \mathrm{mg} / \mathrm{kg} / \mathrm{día})$ y la duración del tratamiento es corta (dosis total acumulada media de $30-90 \mathrm{mg} / \mathrm{kg})^{45,48}$.
El acné neonatal es una dermatosis transitoria que rara vez indica un problema médico latente. Habitualmente se autolimita en los primeros meses de la vida. Cuando la expresión clínica sea grave y prolongada, se deben buscar causas endocrinas de virilización.

\section{Responsabilidades éticas}

Protección de personas y animales. Los autores declaran que para esta investigación no se han realizado experimentos en seres humanos ni en animales.

Confidencialidad de los datos. Los autores declaran que han seguido los protocolos de su centro de trabajo sobre la publicación de datos de pacientes.

Derecho a la privacidad y consentimiento informado. Los autores declaran que en este artículo no aparecen datos de pacientes. 


\section{Conflicto de intereses}

Los autores declaran no tener ningún conflicto de intereses.

\section{Financiamiento}

Ninguno.

\section{Bibliografía}

1. Wagner A. Distinguishing vesicular and pustular disorders in the neonate. Curr Opin Pediatr. 1997;9:396-405.

2. Giknis FL, Hall WK, Tolman MM. Acne neonatorum. AMA Arch Derm Syphilol. 1952;66:717-21.

3. Katsambas AD, Katoulis AC, Stavropoulos P. Acne neonatorum: a study of 22 cases. Int J Dermatol. 1999;38:128-30.

4. Antoniou C, Dessinioti C, Stratigos AJ, Katsambas AD. Clinical and therapeutic approach to childhood acne: an update. Pediatr Dermatol. 2009;26:373-80.

5. Friedlander SF, Baldwin HE, Mancini AJ, Yan AC, Eichenfield LF. The acne continuum: an age-based approach to therapy. Semin Cutan Med Surg. 2011;30(Suppl 3):S6-S11.

6. Jansen T, Burgdorf WH, Plewig G. Pathogenesis and treatment of acne in childhood. Pediatr Dermatol. 1997;14:17-21.

7. Cantatore-Francis JL, Glick SA. Childhood acne: evaluation and management. Dermatol Ther. 2006;19:202-9.

8. Kaminsky A, Florez-White M, Arias MI, Bagatin E. Clasificación del acné: Consenso Ibero-Latinoamericano, 2014. Med Cutan Iber Lat Am. 2015;43:18-23

9. Larralde M, Luna P. Vesiculopustular, bullous and erosive diseases of the newborn. En: Schachner AL, Hansen RC, editores. Pediatric Dermatology. Philadelphia: Elsevier; 2011. p. 328-42.

10. Braun-Falco O, Plewig G, Wolff HH, Winkelmann RK. Diseases of the sebaceous follicules. En: Dermatology. Berlin: Springer; 1991. p. 716-43.

11. Bekaert $C$, Song $M$, Delvigne A. Acne neonatorum and familial hyperandrogenism. Dermatology. 1998;196:453-4.

12. Henderson CA, Taylor J, Cunliffe WJ. Sebum excretion rates in mothers and neonates. Br J Dermatol. 2000;142:110-1.

13. Zouboulis CC. Acne and sebaceous gland function. Clin Dermatol. 2004;22:360-6.

14. Kuiri-Hänninen $T$, Haanpää $M$, Turpeinen $U$, Hämäläinen $E$, Dunkel L, Sankilampi U. Transient postnatal secretion of androgen hormones is associated with acne and sebaceous gland hypertrophy in early infancy. J Clin Endocrinol Metab. 2013;98:199-206.

15. Pochi PE, Strauss JS. Endocrinologic control of the development and activity of the human sebaceous gland. J Invest Dermatol. 1974:62:191-201.

16. Lanciotti L, Cofini M, Leonardi A, Penta L, Esposito S. Up-to-date review about minipuberty and overview on hypothalamic-pituitary-gonadal axis activation in fetal and neonatal life. Front Endocrinol (Lausanne). 2018;9:410.

17. Forest MG, Cathiard AM, Bertrand JA. Evidence of testicular activity in early infancy. J Clin Endocrinol Metab. 1973;37:148-51.

18. Bidlingmaier F, Dörr HG, Eisenmenger W, Kuhnle U, Knorr D. Contribution of the adrenal gland to the production of androstenedione and testosterone during the first two years of life. J Clin Endocrinol Metab. 1986:62:331-5.

19. Lucky AW. Hormonal correlates of acne and hirsutism. Am J Med. 1995;98:89S-94S.

20. Lucky AW. A review of infantile and pediatric acne. Dermatology. 1998;196:95-7.
21. Martín R, Frías M, Blanco A. Hiperandrogenismo. Pediatr Integral 2015;19:498-508.

22. Thigpen AE, Silver RI, Guileyardo JM, Casey ML, McConnell JD, Russell DW. Tissue distribution and ontogeny of steroid 5 alpha-reductase isozyme expression. J Clin Invest. 1993;92:903-10.

23. Agache $P$, Blanc $D$, Barrand $C$, Laurent $R$. Sebum levels during the first year of life. Br J Dermatol. 1980;103:643-9.

24. Wang $\mathrm{H}$, Wang J, Zhou M, Jia Y, Yang M, He C. Prediction of neonatal acne based on maternal lipidomic profiling. J Cosmet Dermatol. 2020;19:2759-66.

25. Bergman JN, Eichenfield LF. Neonatal acne and cephalic pustulosis: is Malassezia the whole story? Arch Dermatol. 2002;138:255-7.

26. Ayhan M, Sancak B, Karaduman A, Arikan S, Sahin S. Colonization of neonate skin by Malassezia species: relationship with neonatal cephalic pustulosis. J Am Acad Dermatol. 2007;57:1012-8.

27. Bernier V, Weill FX, Hirigoyen V, Elleau C, Feyler A, Labrèze C, et al. Skin colonization by Malassezia species in neonates: a prospective study and relationship with neonatal cephalic pustulosis. Arch Dermatol. 2002;138:215-8.

28. Herane MI, Ando I. Acne in infancy and acne genetics. Dermatology. 2003:206:24-8.

29. Mancini AJ, Baldwin HE, Eichenfield LF, Friedlander SF, Yan AC. Acne life cycle: the spectrum of pediatric disease. Semin Cutan Med Surg. 2011;30:S2-S5.

30. Maroñas-Jiménez L, Krakowski AC. Pediatric acne: clinical patterns and pearls. Dermatol Clin. 2016;34:195-202.

31. Alakloby OM, Bukhari IA, Awary BH, Al-Wunais KM. Acne neonatorum in the Eastern Saudi Arabia. Indian J Dermatol Venereol Leprol. 2008;74:298.

32. Rapelanoro R, Mortureux P, Couprie B, Maleville J, Taïeb A. Neonatal Malassezia furfur pustulosis. Arch Dermatol. 1996;132:190-3.

33. Yañes-Díaz S, Martínez-Herrera B, González-López MA. Pustulosis cefálica neonatal por «Malassezia». Acta Pediatr Esp. 2008;66:299-301.

34. Reginatto FP, Villa DD, Cestari TF. Benign skin disease with pustules in the newborn. An Bras Dermatol. 2016:91:124-34

35. Hulsmann AR, Oranje AP. Educational paper: neonatal skin lesions. Eur J Pediatr. 2014;173:557-66.

36. Hernández A. La piel del recién nacido. En: Torrelo A, editor. Dermatología en pediatría general. Madrid: Grupo Aula Médica; 2007. p. 15-38.

37. Guerra KC, Toncar A, Krishnamurthy K. Miliaria. En: StatPearls. Treasure Island (FL): StatPearls Publishing; 2021. Disponible en: https://www. ncbi.nlm.nih.gov/books/NBK537176.

38. Krüger EMM, Sinkos F, Uhry JF, Boni JCB, Okamoto CT, Purin KSM, et al. Dermatoses in the early neonatal period: their association with neonatal, obstetric and demographic variables. Rev Paul Pediatr. 2019:37:297-304

39. Eichenfield LA, Lee PW, Larralde M, Luna P. Neonatal skin and skin disorders. En: Schachner AL, Hansen RC, editores. Pediatric Dermatology. Philadelphia: Elsevier; 2011. p. 299-373.

40. Serna-Tamayo C, Janniger CK, Micali G, Schwartz RA. Neonatal and infantile acne vulgaris: an update. Cutis. 2014;94:13-6.

41. Lizardo G. Acné en la niñez. Honduras Pediátrica. 1999;20:27-9.

42. Stankler L, Campbell AG. Neonatal acne vulgaris: a possible feature of the fetal hydantoin syndrome. Br J Dermatol. 1980;103:453-5.

43. Navarro L. Alteraciones de las glándulas sebáceas y sudoríparas. En: Torrelo A, editor. Dermatología en pediatría general. Madrid: Grupo Aula Médica; 2007. p. 407-24.

44. Kuflik JH, Schwartz RA. Acneiform eruptions. Cutis. 2000;66:97-100.

45. Yeo L, Ormerod AD. Treatment of acne in children. Am J Clin Dermatol. 2014;15:77-86.

46. Que SKT, Whitaker-Worth DL, Chang MW. Acne: kids are not just little people. Clin Dermatol. 2016;34:710-6.

47. Tom WL, Friedlander SF. Acne through the ages: case-based observations through childhood and adolescence. Clin Pediatr (Phila). 2008;47:639-51.

48. Miller IM, Echeverría B, Torrelo A, Jemec GB. Infantile acne treated with oral isotretinoin. Pediatr Dermatol. 2013;30:513-8. 